\title{
Sensing Order Dispersion for Autonomous Cognitive Radios
}

\author{
Zaheer Khan, Janne J. Lehtomäki \\ Centre for Wireless Communications (CWC) \\ University of Oulu, Finland
}

\author{
Miia Mustonen, Marja Matinmikko \\ VTT, Technical Research Center of Finland \\ P.O. Box 1100 FI-90571 Oulu, Finland
}

\begin{abstract}
When autonomous cognitive radios (CRs) have to search multiple potential channels for spectrum opportunities, they face competition from one another to access the channel. The end result of this competition is reduced CR throughput due to collisions among CRs that transmit in the same channel. In this context, the channel sensing order, i.e., the order in which CRs competing for the channels visit those channels, will affect their probability of successful access. This paper presents an investigation into the sensing order dispersion problem, where two or more autonomous CRs attempt to avoid one another by selecting those sensing orders that reduce the likelihood of collisions among one another. We present adaptive strategies that enable the CRs to achieve sensing order dispersion. We evaluate the performance of the presented adaptive strategies in terms of expected time for the autonomous CRs to reach collision-free sensing orders and also in terms of average number of successful transmissions in the network.
\end{abstract}

\section{INTRODUCTION}

A Cognitive network is a collection of wireless devices that collect information about their environment, and intelligently adapt their operating parameters in order to achieve user goals under the constraint of licensed (primary) user protection [1]. In such networks, a cognitive radio (CR) utilizes spectrum opportunistically by monitoring the licensed frequency spectrum to reliably detect primary user (PU) signals and operating whenever the PU is absent. The detection of PU signals can be achieved by: 1) spectrum sensing; 2) the use of geolocation based databases; or 3 ) the combination of both.

In this paper, we consider a distributed time slotted CR network with multiple frequency channels. The CRs use the beginning of each time slot to sense the channels sequentially in some order $(\mathbb{O}$ to find a free channel to transmit on, if one exists. To visit the potential channels sequentially, each CR autonomously chooses a sensing order $\mathbb{O}$ from the set of available sensing orders and accesses the first vacant channel it finds, if one is found. When two or more autonomous CRs simultaneously sense the same channel, find it free from PU activity, and transmit data on the channel at the same time, a collision occurs. In this context, the common goal of the CRs is to autonomously select those sensing orders in which the likelihood of collisions among the CRs is reduced.

This paper makes the following contributions: 1) To enable the autonomous CRs to reduce the likelihood of collisions among one another we propose a $\rho$-sticky adaptive strategy. Adaptations are in the autonomous choice, by CRs, of the channel sensing order $\mathbb{O}$. When the number of CRs is less than or equal to the number of channels the proposed $\rho$-sticky strategy enables the CRs to arrive at collision-free channel sensing orders, i.e., those sensing orders in which two or more CRs never simultaneously sense the same channels and therefore never collide with one another. We compare such a strategy against a randomize after every collision adaptive strategy, in which a new channel sensing order is randomly selected whenever a CR experiences a collision [2]; 2) We evaluate the proposed adaptive strategy in terms of expected time for the autonomous CRs to reach collision-free sensing orders. 3) Using simulations, we compare the performance of different adaptive and non-adaptive strategies in terms of average number of successful transmissions in the network.

\section{A. Related Work}

The opportunistic spectrum access (OSA) problem for the scenario in which multiple potential channels are available has been investigated in [3]-[10]. The work in [7] consider a time slotted system in which a CR first selects a channel to sense and transmits if that channel is free otherwise it stays silent for the entire duration of that time-slot. This work proposed a learning scheme that employs adaptive randomization based on feedback (occurrence of collisions) for the CRs to arrive at orthogonal channels. In contrast with [7], our work considers sequential channel sensing and to improve convergence time to collision-free sensing orders it proposes an adaptive strategy in which an individual CR using a binary flag $b$ maintains state information.

Several policies for optimal selection of channel sensing order are proposed in [6], [11], [12]. However, these policies are either for a single CR scenario or for a two-user CR network in the presence of a coordinator. Unlike [11], [12], our work takes into account competition for channels among multiple autonomous CRs. A channel sensing order policy for distributed CR networks is proposed in [5]. Unlike [5], our work does not assume knowledge of the channel gains. Moreover, to improve the average number of successful transmissions in the network we propose adaptive strategies that enable the CRs to arrive at collision-free sensing orders.

Learning-based medium access control (MAC) algorithms that discover collision-free schedules are proposed for traditional wireless networks in [13], [14]. However the proposed algorithms cannot be applied straightforwardly to distributed CR networks due to the following reasons: 1) The proposed algorithms do not consider the possibility that due to the reappearance of primary users the availability of channels can vary over time; and 2) They also do not consider that to ensure protection to the primary users from CR interference, the transmitter must perform sensing before transmission to determine which channels are available. 


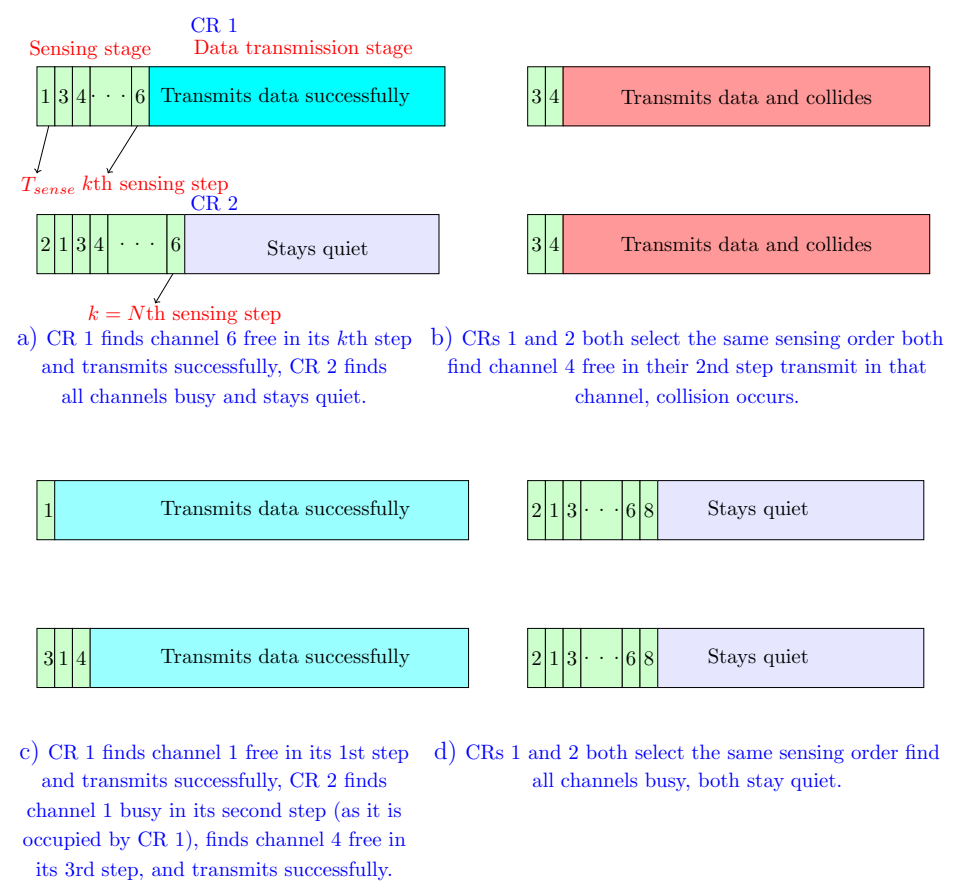

Fig. 1. Different scenarios for sequential channel sensing using sensing orders. In scenarios a) and c) the two CRS are assumed to select different sensing orders, in scenarios b) and d) CRs 1 and 2 are assumed to select the same sensing orders.

\section{SYSTEM MODEL}

We consider a network consisting of $M$ autonomous CRs (transmitter/receiver pairs) with a set of channels $\mathbf{N}=$ $\{1,2, \ldots, N\}$. Each of $M$ CRs is allowed to access one of these channels when it is not occupied by a PU. Due to hardware limitations, a CR can sense only one channel at a time. Also, at any given time each CR can either sense or transmit, but not both. Similar to [3], [7], [8], we assume that the primary users and CRs both use a time slotted system, and each primary user is either present for the entire time slot, or absent for the entire time slot. The channel availability statistics for each channel are assumed to be unknown to the CRs. In each channel, the probability of the PU being present in a given time slot is $\theta_{i}$. Similar to [3], [7], [8] we assume that for each channel, the PU activity (present or absent) in a time slot is independent of the PU activity in other time slots and is also independent of the PU activity in any other channel in any slot. The status of the $i$ th channel in time slot $n$ is a binary variable, $B_{i}(n) \in\{0,1\}$, where 1 means the channel is occupied by a $\mathrm{PU}$ and 0 means the channel is free of a PU activity. Sensing observations are assumed to be perfect as in [7], [8] (In the future, we will explore the effects of the sensing inaccuracies on the performance of the proposed adaptive OSA strategies).

The beginning of each time slot is used by the CRs to sense the potential channels sequentially in some order $\mathbb{O}$ (as explained in Section III) to find a channel that is free of PU (or other CR) activity. We refer to this as the sensing stage. The sensing stage in each slot is divided into a number of sensing steps (see Fig. 1, scenario a)). Each sensing step is used by a $\mathrm{CR}$ to sense a channel. If a CR finds a channel free in its $k$ th sensing step, it transmits in that channel until the end of the slot (data transmission stage) otherwise it stays silent for the entire duration of that time-slot.

Let $Y$ be a random variable representing the number of sensing steps within a time slot until a CR is successful in finding a channel free from PU and other CR activity (given that the CR is successful). Note that, with a constant time slot of duration $T$, the duration of successful data transmission in each slot is a function of $Y$. At the end of each slot, the instantaneous throughput achieved by an autonomous CR is given by

$$
C(\mathrm{Y})=\left\{\begin{array}{lc}
\left(1-\frac{\mathrm{YT}_{\text {sense }}}{T}\right) R, & \text { if successful } \\
0 & \text { otherwise }
\end{array}\right.
$$

where $T_{\text {sense }}$ is the time required to sense each channel and $R$ represents the transmission rate of a CR to its receiver when the channel is available. Note that if the number of potential channels $N$ is larger than $T / T_{\text {sense }}$, then the CR does not have time to visit all channels within a time slot. However, for simplicity and also for practical reasons, we assume throughout the paper that $N<T / T_{\text {sense }}$. Figure 1 illustrates examples of different scenarios for sequential channel sensing using sensing orders.

When autonomous CRs have to search multiple potential channels for spectrum opportunities, they face competition from one another to access the channel. The end result of this competition is reduced CR throughput due to collisions among CRs that transmit in the same channel. In this paper, we are interested in OSA strategies that maximize the average number of successful transmissions in a given slot for the distributed 
CR network (under the PU protection constraint). Note that a successful transmission in a given slot means that a CR finds a channel free from the PU activity and is the sole CR to transmit in that channel.

\section{OSA STRATEGIES}

In this section, we present adaptive OSA strategies that reduce the likelihood of collisions among the autonomous CRs. Adaptations are in the autonomous choice, by CRs, of the channel sensing order $\mathbb{O}$. When the number of CRs is less than or equal to the number of channels the presented strategies allow the CRs in a distributed network to autonomously arrive at collision-free sensing orders, i.e., those sensing orders in which two or more CRs never simultaneously sense the same channels and therefore never collide with one another.

\section{a) $\rho$-sticky strategy:}

We propose a $\rho$-sticky strategy in which a $\mathrm{CR}$ maintains state information using a binary flag $b$. Let $\mathbf{S}$ denote the number of available sensing orders. The sensing order that a CR selects can either come from the space of all permutations of the indices of $N$ channels, or some subset thereof. Let each $\mathrm{CR}$ maintain an $\mathbf{S}$-element probability vector $p$ (i.e., all components are non-negative and add to 1) and let $p_{i}$ represents the probability of choosing the $i$ th sensing order. The $\rho$-sticky strategy involves the following steps.

1) Initialize $p=\left[\frac{1}{\mathbf{S}}, \frac{1}{\mathbf{S}}, \ldots, \frac{1}{\mathbf{S}}\right]$ and set the binary flag to $b=0$. 2) Toss a weighted coin to select a sensing order, with $p_{i}$ the probability of choosing sensing order $i$. Sense the channels sequentially in the order given in the selected sensing order.

3) One of three possibilities occurs:

a) Successful transmission: On a successful transmission using the current sensing order $i$, the CR updates $p_{i}$ and $p_{j}$ as $p_{i}=1$ and $p_{j}=0, \forall j \neq i$,

i.e., it utilizes the same sensing order to visit the channels in the next slot.

The CR then sets $b=1$.

b) $C R$ finds all channels busy: On using sensing order $i$ in the current slot if all the channels visited by the CR are currently found busy, i.e., the channels are either occupied by a PU or by another CR, the CR updates $p_{i}$ and $p_{j}$ as $p_{i}=1$ and $p_{j}=0, \forall j \neq i$, i.e., it utilizes the same sensing order to visit the channels in the next slot.

c) $C R$ collides with another $C R$ : On experiencing a collision in the current slot using sensing order $i$, the CR updates $p_{i}$ as

$$
p_{i}= \begin{cases}\rho & \text { if } b=1 \\ 1 / \mathbf{S} & \text { otherwise }\end{cases}
$$

and updates $p_{j}$ as

$$
p_{j}= \begin{cases}\frac{1-\rho}{\mathbf{S}-1} & \text { if } b=1 \\ 1 / \mathbf{S} & \text { otherwise }\end{cases}
$$

where $\rho$ is a design parameter and $0<\rho<1$.

The CR then sets $b=0$.

i.e., on experiencing a collision in the current slot, if $b=1$ a CR sticks with the current sensing order with probability $\rho$, and if $b=0$ it randomly, with equal probability, selects a new sensing order. Moreover, setting of $b=0$ allows a CR to restart checking for a successful transmission using a sensing order.

4) Return to 2 .
The detection of a collision is implemented in the following way. The CR, upon transmitting in a free channel in the $n$th slot, receives an acknowledgement (ACK) on whether the transmission in that slot was successful. If an ACK is not received then the $\mathrm{CR}$ concludes that a collision occurred.

\section{Intuition for the $\rho$-sticky strategy:}

i) Benefit of setting $b=1$ after success:

In the $\rho$-sticky strategy whenever a CR experiences a collision using a sensing order $i$, a CR using the binary flag $b$ takes into account whether it was successful in the previous slots using that sensing order. If it was successful it sets $b=1$ and after experiencing a collision sticks to the sensing order $i$ with probability $\rho$. This stickiness improves the speed of convergence to collision-free sensing orders as successful CRs tend to stick with their sensing orders, reducing the number of CRs randomly selecting the sensing orders.

ii) Motivation for setting $b=0$ after a collision:

Let us assume that before experiencing a collision a $\mathrm{CR}$ was successful using a sensing order $i$, i.e., binary flag $b$ is set to 1. After experiencing a collision, in the next time slot it may happen that the CR selects with probability $1-\rho$ some other sensing order $j, j \neq i$. In this scenario if $b=1$ then the CR will wrongly believe that it was successful using the sensing order $j$ in the previous time slots. To avoid this, the CRs reset the value of $b$ to 0 after experiencing a collision (see step $3 c$ of $\rho$-sticky strategy).

\section{Convergence of $\rho$-sticky strategy:}

For $N \geq M$, we can show that from colliding sensing orders, the CRs will arrive at collision-free sensing orders in a finite number of time slots.

Assume that in the $n$th time slot the network starts from colliding sensing orders. When the network is in colliding sensing orders then there is non-zero probability of collision between two or more CRs that have picked the colliding sensing order in the $n$th time slot. Given that this collision happens there is a non-zero probability that colliding CRs pick different sensing orders in time slot $n+1$. If in time slot $n+1$, all CRs have collision-free sensing orders then the network has converged. However, if the network is still in colliding sensing orders then there is again a non-zero probability of collision between two or more CRs that have picked the colliding sensing order in the previous slots. This continues until the network arrives at collision-free sensing orders (which will take finite number of time slots due to non-zero probability of arriving at collision-free sensing orders from colliding sensing orders).

b) Randomize after every collision strategy:

We compare the proposed $\rho$-sticky strategy against the randomize after every collision strategy (first proposed by us in [2]). In this strategy, initially each CR randomly selects a sensing order form the set of available sensing orders. In the 


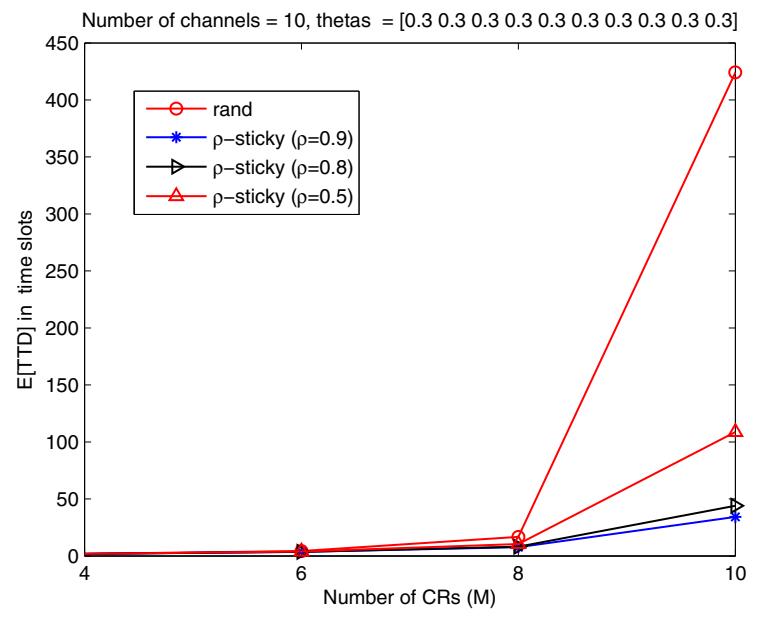

Fig. 2. Simulated expected TTD of the randomize after every collision (rand) strategy and $\rho$-sticky strategy. Number of CRs $M$ varied between 4 to 10 and $N$ is fixed at 10 channels.

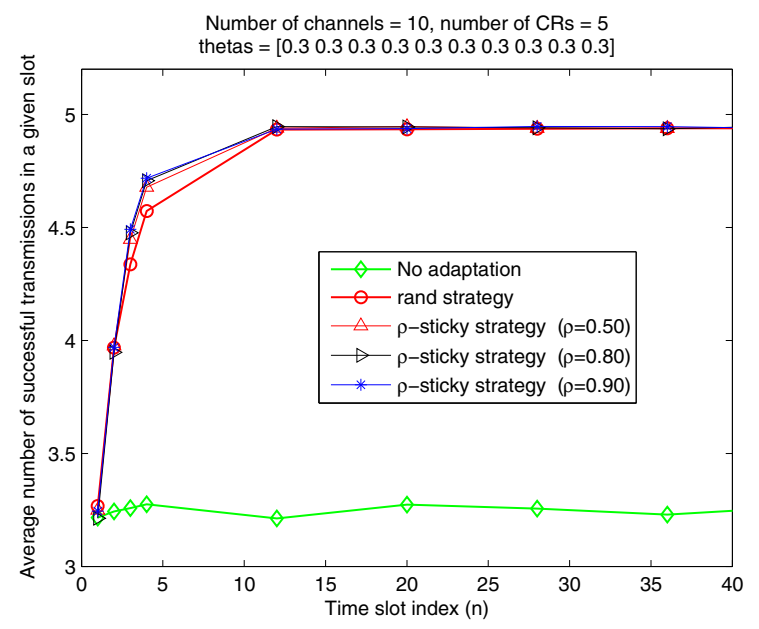

Fig. 3. Simulation results for the average number of successful transmissions for the CR network in a given time slot for different OSA strategies. Number of CRs $M$ is fixed at 5 and number of channels $N$ is fixed at 10 .

next time slots, a CR randomly, with equal probability, selects a new sensing order only if it has experienced a collision in the previous slot; otherwise, it retains the previously selected sensing order.

c) OSA strategy without adaptation:

In each time slot, a sensing order (from the set of available sensing orders) is randomly selected (with equal probability) by each CR independently and then channels are sensed by each $\mathrm{CR}$ according to its own sensing order.

\section{PERformance AnAlysis OF THE $\rho$-STICKy STRATEGY}

Our previous work in [2] showed that when adaptation is employed, the CRs converge quickly to collision-free channel sensing orders when they select sensing orders from a

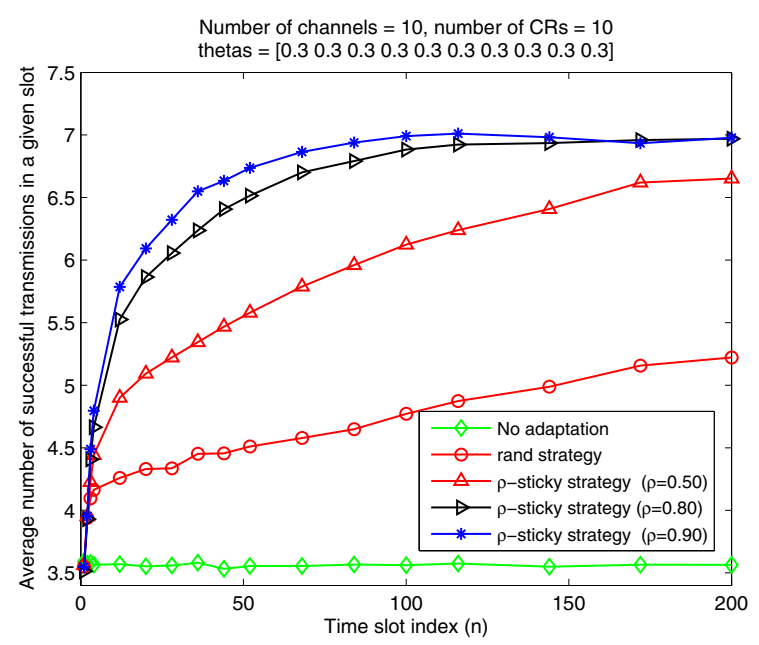

Fig. 4. Simulation results for the average number of successful transmissions for the CR network in a given time slot for different OSA strategies. Number of CRs $M$ and number of channels $N$ is fixed at 10 .

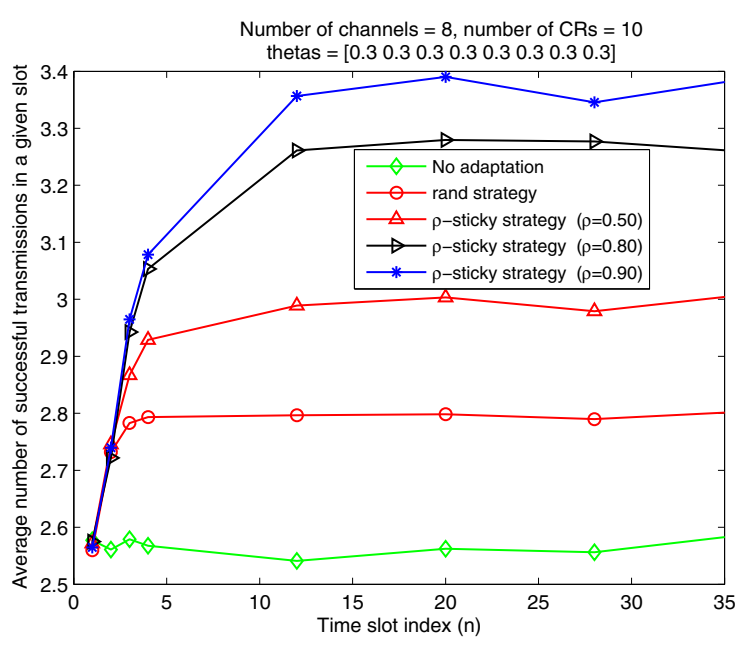

Fig. 5. Simulation results for the average number of successful transmissions for the CR network in a given time slot for different OSA strategies. Number of CRs $M$ is fixed at 10 and number of channels $N$ is fixed at 8 .

predefined Latin Square $\Phi$ as compared to when they select sensing orders from the space of all permutations of $N$ channels. Therefore, to evaluate the performance of the proposed strategy we assume that each CR selects sensing orders from a predefined Latin Square (selects a row of a Latin square), where a Latin Square is an $N$ by $N$ array of $N$ channel indices in which every channel index occurs exactly once in each row and column of the array. For example, with $|\mathbf{N}|=5$, there are many different Latin Squares and one example out of many is given as:

$$
\Phi=\left(\begin{array}{lllll}
1 & 2 & 3 & 4 & 5 \\
5 & 1 & 2 & 3 & 4 \\
4 & 5 & 1 & 2 & 3 \\
3 & 4 & 5 & 1 & 2 \\
2 & 3 & 4 & 5 & 1
\end{array}\right)
$$


We are interested in strategies that: 1) Minimize the expected time to dispersion (E[TTD]), i.e., the expected time (typically measured in time slots) for the autonomous CRs to reach collision-free sensing orders; and 2) Maximize the average number of successful transmissions in a given slot for the distributed CR network.

We simulate a distributed CR network comprising $M$ CRs and $N$ channels. The probability of the PU being present in a given time slot for all $N$ channels is set to $\theta=0.3$. To analyze the impact of parameter $\rho$ on the performance of the $\rho$-sticky strategy, we also vary the value of parameter $\rho$ between 0.5 to 0.9 .

\section{a) Performance in terms of E[TTD]:}

For $N=10$ channels and $M \leq N$, the expected TTD for the randomize after collision strategy using a Latin Square (rand) and the $\rho$-sticky strategy using a Latin Square ( $\rho$-sticky) are plotted in Fig. 2. It can be seen in Fig. 2 that the $\rho$-sticky strategy takes on average either equal or less number of time slots than the rand strategy. It can also be seen in Fig. 2 that the $\rho$-sticky strategy on average requires the less number of time slots when the value of $\rho$ is set to 0.9 , as compared to when the value of $\rho$ is set to 0.5 or 0.8 . We note that the optimal value of parameter $\rho$ may change when the effects of sensing inaccuracies are taken into account. One of the extensions we envision for our current research is the consideration of the effects of sensing inaccuracies on the choice of parameter $\rho$.

b) Performance in terms of average number of successful transmissions:

\section{i) Case 1 (When $N \geq M$ ):}

In Figs. 3 and 4, for $N=10$ channels we compare the performance of the proposed strategies in terms of the average number of successful transmissions in a given slot in the CR network under two different scenarios, when $M<N$ and when $M=N$. The two figures (Figs. 3 and 4) show that for both scenarios the $\rho$-sticky strategy outperforms the rand strategy. Particularly for the scenario when $M=N \rho$-sticky strategy significantly outperforms the rand strategy (see Fig. 4). It can also be seen in Figs. 3 and 4, that the $\rho$-sticky strategy maximizes the average number of successful transmissions in the network when the value of $\rho$ is set to 0.9 .

ii) Case 2 (When $N<M$ ):

It can be seen in Fig. 5 that when the number of autonomous CRs is greater than the number of channels $N$, the proposed $\rho$-sticky strategy with $\rho=0.9$ maximizes the average number of successful transmissions in the network (Fig. 5). Note that when $N<M$ arrival at collision-free sensing orders is not possible.

\section{Conclusions and Future Research}

In this research we show how autonomous CRs can avoid collisions by using adaptive opportunistic spectrum access strategies. We propose a $\rho$-sticky adaptive strategy that enable the CRs to reduce the likelihood of collisions among one another. When the number of CRs is less than or equal to the number of channels the proposed $\rho$-sticky strategy enables the CRs to arrive at collision-free channel sensing orders. We show that the proposed $\rho$-sticky strategy minimizes expected TTD and maximizes the average number of successful transmissions in the network as compared to the randomize after every collision adaptive strategy.

In our future research, we will extend this work to explore the impact of imperfect information (sensing errors and collision detection inaccuracies) on the performance of the proposed adaptive OSA strategy.

\section{REFERENCES}

[1] R. W. Thomas, L. A. DaSilva, and A. B. MacKenzie, "Cognitive networks," in Proceedings of the IEEE International Dynamic Spectrum Access Networks (DySPAN), 2005, pp. 352 -360.

[2] Z. Khan, J. J. Lehtomäki, L. A. DaSilva, and M. Latva-aho, "Analysis of autonomous opportunistic spectrum access strategies for cognitive radios: Benefits of adaptive strategies," in University of Oulu, Tech. Rep., Dec., 2010.

[3] R. Fan and H. Jiang, "Optimal multi-channel cooperative sensing in cognitive radio networks," IEEE Transactions on Wireless Communications, vol. 9, no. 3, pp. 1128-1138, Mar. 2010.

[4] — "Channel sensing-order setting in cognitive radio networks: a twouser case," IEEE Transactions on Vehicular Technology, vol. 58, no. 9, pp. 4997-5008, Nov. 2009.

[5] H. T. Cheng and W. Zhuang, "Simple channel sensing order in cognitive radio networks," IEEE Journal on Selected Areas in Communications, vol. 29 , no. 4, 2011.

[6] N. B. Chang and M. Liu, "Competitive analysis of opportunistic spectrum access strategies," in Proceedings of the IEEE International Conference on Computer Communications (INFOCOM), Phoenix, AZ, USA, Apr. 2008, pp. 1535-1542.

[7] A. Anandkumar, N. Michael, A. K. Tang, and A. Swami, "Distributed learning and allocation of cognitive users with logarithmic regret," IEEE Journal on Selected Areas in Communications, submitted, 2010. [Online]. Available: http: //www.mit.edu/ animakum/pubs/AnandkumarJSAC10.pdf

[8] H. Li, "Multi-agent $Q$-learning for Aloha-like spectrum access in cognitive radio systems," EURASIP Journal on Wireless Communications and Networking, vol. 2010, pp. 1-15, Apr. 2010.

[9] K. Liu and Q. Zhao, "Distributed learning in multi-armed bandit with multiple players," IEEE Transactions on Signal Processing, vol. 58, no. 11 , pp. 5667-5681, Nov. 2010.

[10] H. Liu, B. Krishnamachari, and Q. Zhao, "Cooperation and learning in multiuser opportunistic spectrum access," in Proceedings of the IEEE International Conference on Communications Workshops (ICC workshops), 2008, pp. $487-492$.

[11] N. B. Chang and M. Liu, "Optimal channel probing and transmission scheduling for opportunistic spectrum access," in Proceedings of the Second Workshop on Information Theory and Applications, 2007, pp. 27-38.

[12] H. Jiang, L. Lai, R. Fan, and H. V. Poor, "Optimal selection of channel sensing order in cognitive radio," IEEE Transactions on Wireless Communications, vol. 8, no. 1, pp. 297-307, Jan. 2009.

[13] J. Barceló, B. Bellalta, C. Cano, and M. Oliver, "Learning-BEB: Avoiding collisions in WLAN," in Proceedings of the EUNICE Summer School, 2008.

[14] M. Fang, D. Malone, K. R. Duffy, and D. J. Leith, "Decentralised learning MACs for collision-free access in WLANs," Sep. 2010. [Online]. Available: http://arxiv.org/abs/1009.4386v1 